\title{
METODOLOGIA PARA OBTENÇÃO DO HIDROGRAMA DE ESCOAMENTO SUPERFICIAL EM ENCOSTAS E CANAIS. PARTE I: DESENVOLVIMENTO E AVALIAÇÃO
}

\author{
JOSÉ M. A. SILVA ${ }^{1}$, FERNANDO F. PRUSKI ${ }^{2}$, DEMETRIUS D. SILVA ${ }^{3}$, \\ ROBERTO A. CECÍLIO ${ }^{4}$
}

\begin{abstract}
RESUMO: Desenvolveu-se uma metodologia que permite obter o hidrograma de escoamento superficial e a vazão máxima para qualquer posição ao longo de uma encosta e para seções transversais de canais utilizando o modelo de ondas cinemáticas. A área da encosta é dividida num sistema matricial composto por 100 linhas e 100 colunas. Na encosta, considera-se que o escoamento ocorre na direção da declividade e que a vazão de cada pixel é a soma da vazão produzida nesse com a vazão advinda dos pixels que contribuem com o escoamento superficial para o pixel em análise. No canal, a vazão é calculada pela soma dos hidrogramas advindos das colunas do sistema reticulado. A comparação entre os valores de lâmina e vazão máxima de escoamento superficial obtidas experimentalmente e calculadas em duas condições (encosta e bacia) permitiu evidenciar que a metodologia, comparada aos métodos Racional e do Número da Curva, ofereceu boas estimativas tanto da lâmina quanto da vazão máxima de escoamento superficial.
\end{abstract}

PALAVRAS-CHAVE: modelagem hidrológica, conservação de água e solo.

\section{METHODOLOGY FOR OBTENTION OF SUPERFICIAL FLOW HYDROGRAPH IN HILLSIDES AND CANALS. PART I: DEVELOPMENT AND EVALUATION}

\begin{abstract}
It was developed a methodology that allows obtaining the hydrograph of superficial flow and maxim flow of any position along a hillside and transversal sections of canals. The area of the hillside is divided in a matrix system having 100 lines and 100 columns. It was considered that the flux occurs in the direction of the hillside's slope and the flow of each pixel is the addition between its own flow and the flows that come from the pixels that contribute to the superficial flow in the pixel under analysis. In the canal the flow is taken as the addition of the hydrographs that come from the columns in the reticular system. By the new methodology the comparison of the total volume and maximum surface runoff flow values, obtained in two experimental conditions (hillside and hydrographic basin), allowed to conclude that the new model provided good performance when compared to the Rational Method and the Curve Number Method.
\end{abstract}

KEYWORDS: hydrologic modeling; soil and water conservation.

\section{INTRODUÇÃO}

Das fases associadas ao ciclo hidrológico, provavelmente, a mais importante para as obras de engenharia é o escoamento superficial, que corresponde ao segmento relacionado ao deslocamento de água sobre a superfície do solo. Além das partículas de solo em suspensão, o escoamento superficial transporta nutrientes, matéria orgânica, sementes e defensivos agrícolas que, além de causarem prejuízos à produção agropecuária, também causam poluição nos cursos d'água (PRUSKI et al., 2003).

\footnotetext{
${ }^{1}$ Engo Civil, Doutor em Engenharia Agrícola, DEA/UFV, Viçosa - MG, Fone: (0XX31) 3899-1912, jmarcio@ gprh.ufv.br

${ }^{2}$ Eng ${ }^{0}$ Agrícola, Prof. Titular, DEA/UFV, Bolsista do CNPq, ffpruski@ufv.br

${ }^{3}$ Eng $^{\text {o }}$ Agrônomo, Prof. Adjunto, DEA/UFV, Bolsista do CNPq, david@ufv.br

${ }^{4}$ Engo Agrícola, Doutor em Engenharia Agrícola, DEA/UFV, rcecilio@ vicosa.ufv.br

Recebido pelo Conselho Editorial em: 5-10-2005
}

Aprovado pelo Conselho Editorial em: 5-9-2006 
Informações a respeito do volume de escoamento superficial são necessárias em estudos relacionados ao manejo da água e do solo, à eficiência dos métodos de preparo e cultivo do solo e ao planejamento de irrigação (PATHAK et al., 1989) ou quando se deseja construir estruturas capazes de armazenar água. No dimensionamento de obras hidráulicas, em que o objetivo é a condução do excesso de água para fora da área de interesse, o conhecimento da vazão máxima de escoamento superficial torna-se fundamental (SCHWAB et al., 1966).

Medições do escoamento superficial no campo são caras e demandam tempo, e isso tem motivado o desenvolvimento e o uso de modelos matemáticos para predizer o escoamento superficial (MA et al., 1998). Dentre os métodos para estimar as vazões máximas de escoamento superficial, destacam-se: o método racional, o método de Cook, o Hidrograma Unitário e a fórmula de Mac Math. Dentre os métodos para calcular o volume total escoado superficialmente, destacamse o do Balanço Hídrico Diário e o Método do Número da Curva, e para ambos os métodos, o conhecimento da chuva de projeto e o da capacidade de infiltração da água no solo são requeridos (PRUSKI et al., 1997).

Estudo realizado pelo "Water Resources Council", citado por BONTA \& RAO (1992), documenta a dificuldade de aplicar os procedimentos para estimativa da vazão máxima de escoamento superficial, em razão da imprecisão de alguns dos métodos costumeiramente usados e da grande subjetividade nessas estimativas. Em vista do exposto, a investigação de um método que produza estimativas confiáveis de vazão máxima de escoamento superficial torna-se fundamental para que se possa fazer correto dimensionamento de obras hidráulicas.

Neste trabalho, teve-se o objetivo de desenvolver e validar uma metodologia que permita obter, para qualquer precipitação, o hidrograma de escoamento superficial para qualquer ponto localizado em uma encosta ou nas seções transversais do canal de terraços ou drenos de superfície.

\section{MATERIAL E MÉTODOS}

No desenvolvimento do modelo, denominado HIDROGRAMA, foram consideras duas condições distintas: a encosta e o canal, e para o traçado do hidrograma de escoamento superficial, partiu-se do princípio de que a vazão aumenta até o momento em que a contribuição advinda do ponto mais remoto atinge a seção considerada e, a partir daí, a vazão decresce com o tempo. Dessa forma, são identificados dois trechos distintos no traçado do hidrograma de escoamento superficial (Figura 1):

- trecho ascendente: há crescimento da vazão com o tempo em virtude do aumento da área de contribuição para o escoamento superficial até a célula considerada, e

- trecho descendente: a vazão decresce com o tempo, começando no momento que a água advinda da célula mais remota atinge a célula considerada.

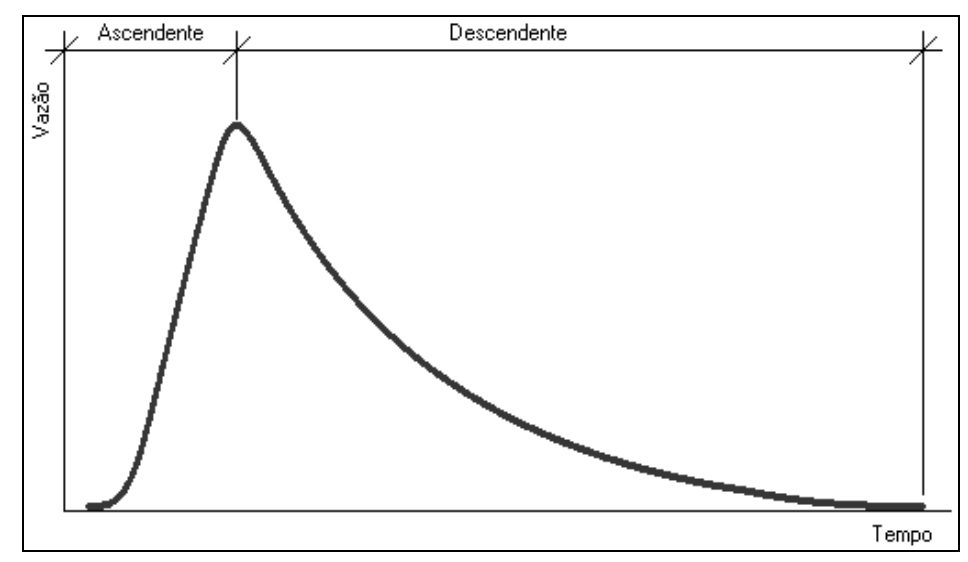

FIGURA 1. Representação gráfica do hidrograma de escoamento superficial. 
Para o desenvolvimento do modelo, foram estabelecidas as seguintes premissas:

- precipitação uniforme na área analisada e equação de intensidade-duração-freqüência da precipitação (IDF) conhecida para a localidade de interesse;

- solo com umidade próxima à saturação, ou seja, durante a ocorrência da chuva de projeto considera-se o solo na capacidade de campo e a taxa de infiltração da água próxima da taxa de infiltração estável $\left(\mathrm{T}_{\mathrm{ie}}\right)$;

- evaporação nula durante a precipitação, visto tratar-se de precipitações intensas e a umidade do ar ser alta durante a ocorrência da chuva, e

- $\mathrm{T}_{\mathrm{ie}}$ no canal considerada constante durante a acumulação de água nesse.

Para a obtenção do hidrograma de escoamento superficial, subdivide-se a encosta num sistema matricial composto por linhas e colunas. Cada pixel desse sistema matricial possui uma posição identificada pela linha e coluna nas quais se encontra. Na Figura 2a, visualiza-se a subdivisão da área, composta por 10 linhas e 10 colunas, e o canal do terraço ou dreno de superfície. A análise do hidrograma é realizada separadamente para a encosta e para o canal (Figura 2b).
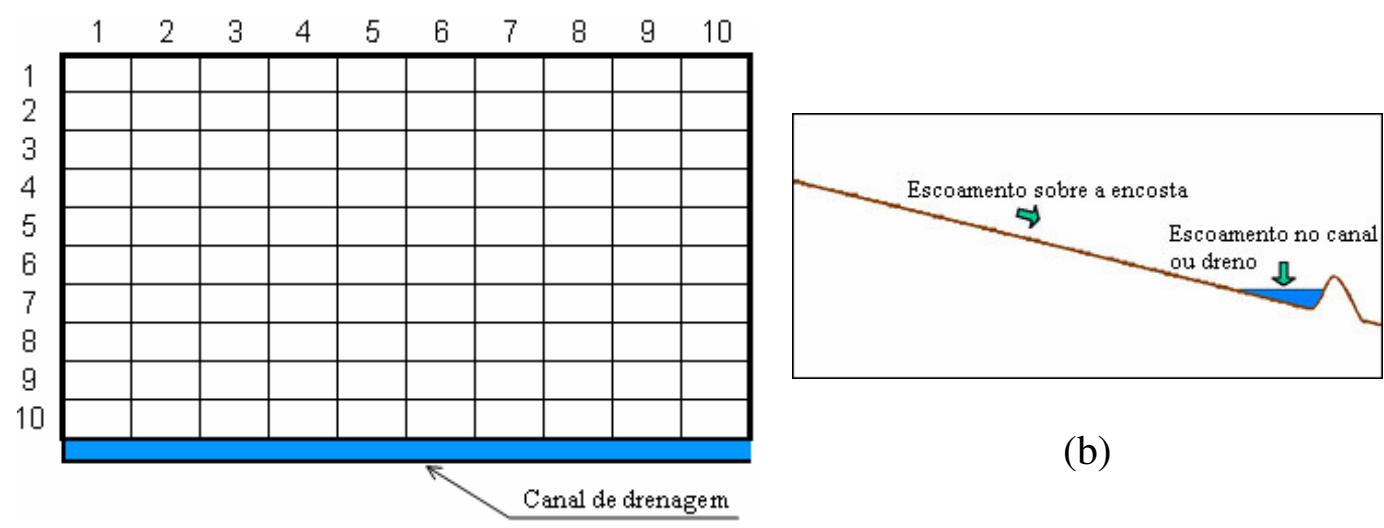

(b)

(a)

FIGURA 2. Representação da subdivisão da área de interesse em um sistema matricial.

\section{Obtenção do hidrograma de escoamento superficial na encosta}

Para essa condição, considera-se que o escoamento superficial ocorre exclusivamente na direção da linha de maior declividade do terreno.

O escoamento superficial inicia-se após o preenchimento das abstrações iniciais (Ia, em mm), cujos valores são calculados pelo Método do Número da Curva, utilizando a equação recomendada pelo USDA-SCS (1985), expressa por:

$$
\mathrm{Ia}=50,8\left(\frac{100}{\mathrm{CN}}-1\right)
$$

em que, CN é o número da curva, cujo valor pode variar entre 1 e 100, e depende do uso e manejo da terra, grupo de solo, condição hidrológica e umidade antecedente do solo.

Como a taxa de infiltração se aproxima da $\mathrm{T}_{\mathrm{ie}}$, considera-se que, quando a precipitação ocorre, a umidade do solo é a máxima definida pelo Método do Número da Curva (AMC III), isto é, a precipitação acumulada durante os cinco dias anteriores à chuva estudada é igual ou maior que $52,5 \mathrm{~mm}$.

O tempo correspondente à ocorrência de Ia é obtido pela eq.(2).

$$
\mathrm{Ia}=\int_{0}^{\mathrm{t}_{\mathrm{Ia}}} \mathrm{i}_{\mathrm{i}} \mathrm{dt}
$$


em que,

$\mathrm{t}_{\mathrm{Ia}}$ - intervalo de tempo entre o início da chuva e o início do escoamento superficial, min, e $\mathrm{i}_{\mathrm{i}}$ - intensidade instantânea de precipitação, $\mathrm{mm} \mathrm{h}^{-1}$.

A intensidade de precipitação instantânea é obtida com a eq.(3).

$$
\mathrm{i}_{\mathrm{i}}=\mathrm{i}_{\mathrm{m}}\left(1-\frac{\mathrm{ct}}{\mathrm{t}+\mathrm{b}}\right)
$$

em que,

$\mathrm{i}_{\mathrm{m}}$ - intensidade média máxima de precipitação, $\mathrm{mm} \mathrm{h}^{-1}$, e

b, c - parâmetros adimensionais relativos à localidade.

Para a obtenção de $\mathrm{i}_{\mathrm{m}}$, é utilizada a IDF, cuja forma é:

$$
i_{m}=\frac{K T^{a}}{(t+b)^{c}}
$$

em que,

$\mathrm{T}$ - período de retorno, anos;

$\mathrm{t}$ - duração da precipitação, min, e

$\mathrm{K}, \mathrm{a}, \mathrm{b}, \mathrm{c}$ - parâmetros adimensionais relativos à localidade.

Substituindo a eq.(4) na eq.(2), pode-se obter $t_{\text {Ia }}$ pela eq.(5).

$$
\mathrm{t}_{\mathrm{Ia}}=\frac{60 \mathrm{I}_{\mathrm{a}}\left(\mathrm{t}_{\mathrm{Ia}}+\mathrm{b}\right)^{\mathrm{c}}}{\mathrm{K} \mathrm{T}^{\mathrm{a}}}
$$

Para a resolução dessa equação, é utilizado o método de Newton-Raphson.

A modelagem do escoamento superficial é realizada utilizando o modelo de ondas cinemáticas. As duas equações que regem o escoamento gradualmente variável em superfícies livres foram estabelecidas em 1871, por Saint-Venant. São equações que traduzem os princípios físicos da conservação da massa (equação da continuidade) e da conservação da quantidade de movimento (equação da dinâmica) (SILVA, 1996). O modelo de ondas cinemáticas, uma das formas de aplicação das equações de Saint-Venant, pode ser expresso por JULIEN et al. (1995) e MUÑOZ-CARRERA \& PARSONS (1999):

$$
\frac{\partial \mathrm{h}}{\partial \mathrm{t}}+\frac{\partial \mathrm{q}}{\partial \mathrm{x}}=\mathrm{i}_{\mathrm{i}}-\mathrm{T}_{\mathrm{ie}} \quad \text { e } \quad \mathrm{S}_{\mathrm{o}}=\mathrm{Sf}
$$

em que,

$\mathrm{h}$ - profundidade do escoamento, $\mathrm{m}$;

$\mathrm{q}$ - vazão por unidade de largura na direção $\mathrm{x}, \mathrm{m}^{2} \mathrm{~s}^{-1}$;

$\mathrm{x}$ - direção do escoamento, $\mathrm{m}$;

$\mathrm{S}_{\mathrm{o}}$ - declividade da superfície do solo, $\mathrm{m} \mathrm{m}^{-1}$, e

$\mathrm{S}_{\mathrm{f}}$ - declividade da linha de energia, $\mathrm{m} \mathrm{m}^{-1}$.

$\mathrm{O}$ modelo de ondas cinemáticas considera a declividade da linha de energia $\left(\mathrm{S}_{\mathrm{f}}\right)$ igual à declividade da superfície do solo $\left(S_{\mathrm{o}}\right)$, assumindo uma seção transversal média de escoamento. Com a utilização de equações tipicamente usadas para condições de regime uniforme, obtém-se a relação entre a vazão e a profundidade de escoamento expressa por:

$$
\mathrm{q}=\alpha \mathrm{h}^{\beta}
$$

em que $\alpha$ e $\beta$, utilizando-se da equação de Manning, podem ser expressos como:

$$
\alpha=\frac{\sqrt{\mathrm{S}_{\mathrm{o}}}}{\mathrm{n}} \quad \mathrm{e} \quad \beta=\frac{5}{3}
$$

sendo $\mathrm{n}$, o coeficiente de rugosidade do terreno. 
A eq.(6) é resolvida utilizando-se do método de diferenças finitas, segundo o algoritmo proposto por BRAZ (1990), e o valor da profundidade do escoamento calculado é transformado em vazão pela eq.(7).

Uma vez que tanto $i_{m}$ como $i_{i}$ diminuem com o aumento de $t$, o escoamento superficial (ES) máximo é aquele correspondente ao instante em que $\mathrm{i}_{\mathrm{i}}$ se iguala à $\mathrm{T}_{\mathrm{ie}}$. Para essa condição, tem-se:

$$
\frac{K T^{a}}{(t+b)^{c}}\left(1-\frac{c t}{t+b}\right)=T_{i e}
$$

O valor de t correspondente ao ES máximo é obtido pelo método de convergência de Newton-Raphson.

\section{Obtenção do hidrograma de escoamento superficial no canal do terraço ou dreno de superfície}

A modelagem do escoamento superficial no canal é realizada utilizando-se da eq.(10), proposta por JULIEN et al. (1995). Essa equação é obtida a partir do modelo de ondas cinemáticas aplicado a uma única direção:

$$
\frac{\partial \mathrm{A}}{\partial \mathrm{t}}+\frac{\partial \mathrm{Q}}{\partial \mathrm{x}}=\mathrm{q}
$$

em que,
A - seção transversal do escoamento, $\mathrm{m}^{2}$, e
$\mathrm{Q}$ - vazão de escoamento no canal, $\mathrm{m}^{3} \mathrm{~s}^{-1}$.

A vazão de escoamento no canal é obtida utilizando-se da equação de Manning, expressa por:

$$
\mathrm{Q}=\frac{1}{\mathrm{n}} \mathrm{A} \mathrm{R}_{\mathrm{h}}^{2 / 3} \mathrm{~S}_{\mathrm{c}}^{1 / 2}
$$

em que,

$\mathrm{R}_{\mathrm{h}}$ - raio hidráulico, $\mathrm{m}$, e

$\mathrm{S}_{\mathrm{c}}$ - declividade longitudinal do canal, $\mathrm{m} \mathrm{m}^{-1}$.

A eq.(10) é resolvida utilizando o método de diferenças finitas, segundo o algoritmo proposto por BRAZ (1990), e o valor da profundidade do escoamento é transformado em vazão pela eq.(11). Para a obtenção da vazão proveniente da encosta, aplica-se a metodologia proposta no item "Obtenção do hidrograma de escoamento superficial na encosta".

\section{Obtenção do volume de escoamento superficial}

Em um hidrograma, o volume de escoamento superficial corresponde à área sob a curva representativa da vazão em função do tempo. Para a obtenção dessa área, procede-se à integração do hidrograma, utilizando, para tanto, a regra dos trapézios.

\section{Validação do modelo}

Para a validação do modelo desenvolvido (HIDROGRAMA), foram utilizados dados referentes à vazão máxima $\left(\mathrm{Q}_{\text {máx }}\right)$ e à lâmina de escoamento superficial (LES) obtidos de dois experimentos distintos, sendo um deles relativo a uma encosta e outro a uma bacia hidrográfica.

Na condição da encosta, os dados de $\mathrm{Q}_{\text {máx }}$ e LES foram medidos durante um ano em parcelas experimentais com dimensões de $11 \mathrm{~m}$ de comprimento por 3,5 m de largura e 15,3\% de declividade, localizadas em Viçosa - MG. As parcelas foram mantidas sem cobertura vegetal e sob preparo convencional no sentido do declive. A taxa de infiltração estável $\left(\mathrm{T}_{\mathrm{ie}}\right)$ da água no solo, nas parcelas experimentais, foi determinada com o simulador de chuvas, sendo igual a $10 \mathrm{~mm} \mathrm{~h}^{-1}$.

Na condição da bacia, os valores de $\mathrm{Q}_{\text {máx }}$ e LES foram medidos por SILVA (2002) durante um ano, em uma bacia hidrográfica experimental com 3,99 ha, também localizada em Viçosa - 
MG. Toda a área da bacia era ocupada por pastagens degradadas, sendo a declividade média igual a $65 \%$. A $\mathrm{T}_{\mathrm{ie}}$ média na bacia, determinada com o simulador de chuvas, foi igual a $60 \mathrm{~mm} \mathrm{~h}^{-1}$. Para a utilização do HIDROGRAMA nessa condição, foi necessário considerar a bacia como a combinação de duas encostas representativas das condições da bacia, com o que os hidrogramas de escoamento superficial calculados para cada uma das encostas foram somados. Do hidrograma resultante, foi obtido o valor calculado para $Q_{\text {máx }}$ A transformação da bacia nas duas encostas equivalentes foi realizada considerando o comprimento do talvegue da bacia como o comprimento do canal. A declividade média considerada para o canal foi a declividade equivalente constante, obtida conforme VILELLA \& MATTOS (1975), e para qual o tempo de percurso da água ao longo da extensão do perfil longitudinal é igual ao tempo de deslocamento correspondente ao perfil real. O comprimento das duas encostas foi calculado de modo que a soma da área de ambas fosse igual à área da bacia. A declividade considerada para cada uma das encostas foi a declividade média das áreas por elas representadas.

Para a aplicação do HIDROGRAMA e dos métodos Racional e do Número da Curva, considerou-se um período de retorno da precipitação (T) igual a um ano, devido ao fato de esse ter sido o período de coleta de dados nos dois experimentos considerados. Nas aplicação das três metodologias, considerou-se a equação de intensidade-duração-freqüência da precipitação ajustada por PINTO (1995), para a cidade de Viçosa - MG.

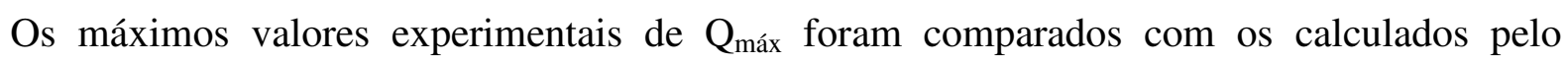
HIDROGRAMA e com os calculados com a utilização do Método Racional. Na aplicação do Método Racional, o tempo de concentração foi calculado pela equação de Kirpich. Os valores do coeficiente de escoamento superficial (C) foram determinados segundo recomendações do Soil Conservation Service - USDA (PRUSKI et al., 2003) como sendo igual a 0,8 para a condição de encosta e 0,6 para a condição de bacia. Os valores do número da curva foram iguais a 96 e 91, para a encosta e para a bacia, respectivamente, considerando AMC III.

Compararam-se, também, os máximos valores de LES medidos nos dois experimentos com aqueles calculados pelo HIDROGRAMA e pelo Método do Número da Curva. As precipitações consideradas no Método do Número da Curva foram aquelas com duração correspondentes a 6; 12 e 24 horas, uma vez que o método não define qual a duração de precipitação capaz de gerar o maior volume de escoamento superficial, sendo essas durações normalmente recomendadas para a estimativa da LES. O enquadramento do solo, nos diferentes grupos, foi feito de acordo com a $\mathrm{T}_{\mathrm{ie}}$, seguindo as recomendações propostas pelo SOIL CONSERVATION SERVICE (1972).

\section{RESULTADOS E DISCUSSÃO}

$\mathrm{Na}$ Tabela 1, apresentam-se os valores de $\mathrm{Q}_{\text {máx }}$ experimentais e os calculados com a utilização do HIDROGRAMA e do Método Racional. Percebe-se que, na condição de encosta, tanto o HIDROGRAMA quanto o Método Racional produziram superestimativas no valor de Qmáx medido em mais de duas vezes e meia, havendo grande proximidade entre o valor calculado pelo HIDROGRAMA e o calculado pelo Método Racional. Essa aproximação se deve ao fato de que o solo da área em questão possui baixa $\mathrm{T}_{\mathrm{ie}}$, apresenta declividade aproximadamente constante ao longo da direção de escoamento, não tem cobertura vegetal e apresenta preparo no sentido do declive, o que fez com que as condições experimentais e, conseqüentemente, as condições simuladas pelo HIDROGRAMA se aproximassem das condições recomendadas para o emprego do Método Racional.

TABELA 1. Valores de vazão máxima $\left(\mathrm{L} \mathrm{s}^{-1}\right)$ de escoamento superficial ( $\left.\mathrm{Q}_{\text {máx }}\right)$ obtidos experimentalmente e calculados pelo HIDROGRAMA e pelo Método Racional.

\begin{tabular}{ccc}
\hline & Encosta & Bacia \\
\hline Valor experimental & 0,29 & 21,0 \\
HIDROGRAMA & 0,74 & 61,3 \\
Método Racional & 0,79 & 283,0 \\
\hline
\end{tabular}


A análise dos dados de precipitação incidente na encosta evidencia que, no período de cinco dias anteriores à ocorrência do evento que gerou a máxima vazão de escoamento superficial, o total precipitado foi de apenas $41 \mathrm{~mm}$, quantidade insuficiente para garantir que o solo estivesse com umidade próxima à de saturação no momento de ocorrência da precipitação. Assim, o valor de $\mathrm{Q}_{\text {máx }}$ não correspondeu à máxima vazão possível de escoamento superficial, havendo a possibilidade de que esse valor fosse maior, caso a umidade do solo estivesse mais elevada. Além disso, o HIDROGRAMA considera que a chuva capaz de causar o maior escoamento superficial possui duração determinada de acordo com a $\mathrm{T}_{\mathrm{ie}}$. Acontece que a chuva crítica, isto é, a chuva com duração capaz de causar o maior escoamento superficial, não ocorreu durante o ano em que foram feitas as medições.

Com relação à condição da bacia hidrográfica, de maneira análoga à condição da encosta,

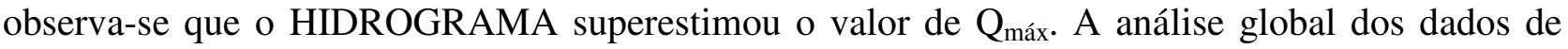
precipitação ocorridos na bacia, nos cinco dias anteriores à ocorrência do evento que gerou o máximo valor de $Q_{\text {máx }}$ (SILVA, 2002), mostra que o total precipitado nesse período foi de apenas $25,3 \mathrm{~mm}$. Do mesmo modo que o ocorrido para a condição da encosta, esse total precipitado não permitiu que a umidade do solo estivesse em um valor próximo à saturação, acarretando, conseqüentemente, menor vazão de escoamento superficial. Somando-se a esse fator, ainda tem-se o fato de que, como na condição da encosta, não ocorreu chuva crítica considerada pelo HIDROGRAMA.

Ainda a respeito da condição da bacia, percebe-se que, apesar da superestimativa feita pelo HIDROGRAMA, o valor de $Q_{\text {máx }}$ calculado promoveu boa estimativa do valor experimental, principalmente quando comparado com o valor calculado com a utilização do Método Racional. Observa-se que o Método Racional promoveu grande superestimativa da vazão de escoamento superficial, comparado tanto com o valor experimental (13,5 vezes) quanto com o calculado pelo HIDROGRAMA (4,6 vezes). Isso ocorre devido ao fato de a bacia, apesar de ter grande declividade média, apresentar alta $\mathrm{T}_{\mathrm{ie}}$, alta rugosidade hidráulica e grande capacidade de armazenamento superficial de água, o que acarreta o retardo do início do processo de escoamento superficial. Como a determinação do coeficiente de escoamento superficial (C) do Método Racional é feita apenas com base na classe textural e na declividade da área, e o cálculo do tempo de concentração também não considerar a real complexidade do deslocamento do escoamento superficial na bacia, o método foi incapaz de calcular um valor de $\mathrm{Q}_{\text {máx }}$ próximo ao valor experimental.

Comparando-se as simulações realizadas para as duas condições, percebe-se grande sensibilidade apresentada pelo HIDROGRAMA à variação da $\mathrm{T}_{\mathrm{ie}}$, uma vez que as diferenças percentuais entre os valores experimentais e os calculados não apresentaram grande variação. Observando-se o grande aumento na diferença percentual apresentada entre os valores de $\mathrm{Q}_{\text {máx }}$ reais e estimados com a utilização do Método Racional, pode-se verificar que esse não apresenta sensibilidade à $\mathrm{T}_{\mathrm{ie}}$, o que já era esperado, uma vez que essa não é levada em consideração para a escolha do coeficiente de escoamento superficial (C). Tal fato corrobora afirmações de diversos pesquisadores de que o Método Racional é reconhecidamente uma metodologia que superestima a vazão máxima de escoamento superficial, desconsiderando a real influência, não só da infiltração da água no solo, como da interceptação pela cobertura vegetal, do armazenamento superficial, do perfil de precipitação e da rugosidade hidráulica da superfície do solo.

Na Tabela 2, são apresentados os valores de LES experimentais e calculados por intermédio do HIDROGRAMA e do Método do Número da Curva, considerando durações da precipitação de 6; 12 e 24 horas. Para a condição de encosta, verifica-se que o HIDROGRAMA superestimou a lâmina de escoamento superficial, o que pode ser justificado pelo fato de que a chuva que gerou o valor experimental da LES não correspondeu à chuva de projeto considerada pelo HIDROGRAMA, isto é, não foi aquela precipitação cuja intensidade e duração são capazes de causar o máximo volume de escoamento superficial para o solo da área $\left(\mathrm{T}_{\mathrm{ie}}=10 \mathrm{~mm} \mathrm{~h}^{-1}\right)$. Comparando-se os valores de LES calculados, observa-se que o HIDROGRAMA estimou um valor 
de LES mais próximo ao valor observado, comparado ao Método do Número da Curva, o que pode ser explicado pelo fato de o Método do Número da Curva não ser capaz de estimar a duração crítica da precipitação, isto é, a duração capaz de causar o maior escoamento superficial.

TABELA 2. Valores de lâmina escoada (LES) obtidos experimentalmente e calculados pelo HIDROGRAMA e pelo Método Racional

\begin{tabular}{ccc}
\hline & Encosta & Bacia \\
\hline Valor experimental & $11,5 \mathrm{~mm}$ & $0,7 \mathrm{~mm}$ \\
HIDROGRAMA & $25,2 \mathrm{~mm}$ & $0,5 \mathrm{~mm}$ \\
Método do Número da Curva $(\mathrm{t}=6 \mathrm{~h})$ & $53,4 \mathrm{~mm}$ & $41,9 \mathrm{~mm}$ \\
Método do Número da Curva $(\mathrm{t}=12 \mathrm{~h})$ & $65,9 \mathrm{~mm}$ & $53,7 \mathrm{~mm}$ \\
Método do Número da Curva $(\mathrm{t}=24 \mathrm{~h})$ & $79,9 \mathrm{~mm}$ & $67,0 \mathrm{~mm}$ \\
\hline
\end{tabular}

Para a condição da bacia, observa-se que o HIDROGRAMA apresentou valor de LES muito próximo ao experimental, entretanto pouco inferior a esse, sendo essa diferença inexpressiva. Comparando-se o resultado experimental e o estimado pelo HIDROGRAMA com aqueles obtidos com o emprego do Método do Número da Curva, percebe-se grande diferença, que se deve ao fato de a $\mathrm{T}_{\mathrm{ie}}$ ser muito alta nessa condição. Como o Método do Número da Curva trabalha com faixas muito amplas de $\mathrm{T}_{\text {ie }}$ para a definição do grupo de solo a ser considerado, a baixa sensibilidade está associada a esse parâmetro, diferentemente do HIDROGRAMA, que trabalha com o valor medido da $T_{\text {ie }}$, razão pela qual apresentou melhores estimativas tanto na condição da encosta quanto na condição da bacia.

De um modo geral, observou-se que o HIDROGRAMA forneceu melhores estimativas dos valores de $\mathrm{Q}_{\text {máx }}$ e LES comparado aos métodos Racional e do Número da Curva, respectivamente, demonstrando aplicabilidade tanto para a condição de encosta quanto para a condição de bacia.

\section{CONCLUSÕES}

A metodologia desenvolvida permite a obtenção do hidrograma de escoamento superficial em encostas e em canais, permitindo a quantificação da lâmina e da vazão máxima de escoamento superficial.

A aplicação do HIDROGRAMA permitiu boa estimativa da vazão máxima de escoamento superficial, principalmente quando comparada com os resultados obtidos pelo Método Racional.

A aplicação do HIDROGRAMA permitiu boa estimativa da lâmina de escoamento superficial, principalmente quando comparada com os resultados obtidos pelo Método do Número da Curva.

\section{REFERÊNCIAS}

BONTA, J.V.; RAO, A.R. Estimating peak flows small agricultural watersheds. Journal of Irrigation and Drainage Engineering, Reston, v.118, n.1, p.122-37, 1992.

BRAZ, R.L. Hydrology: an introduction to hydrologic science. New York: Addison-Wesley Publishing Company, 1990. 643 p.

JULIEN, P.Y.; SAGHAFIAN, B.; OGDEN, F.L. Raster-based hydrologic modeling of spatiallyvaried surface runoff. Water Resources Bulletin, Minneapolis, v.31, n.3, p.523-36, 1995.

MA, Q.L.; WAUCHOPE, R.D.; HOOK, J.E.; JOHNSON, A.W.; TRUMAN,C.C.; DOWLER, C.C.; GASCHO, G.J.; DAVIS, J.G.; SUMNER, H.R.; CHANDLER, L.D. GLEAMS Opus, and PRZM-2 model predicted versus measured runoff form a coastal plain loamy sand. Transactions of the ASAE, St. Joseph, v.41, n.1, p.77-88, 1998. 
MUÑOZ-CARRERA, R.; PARSONS, J.E. VFSMOD - Vegetative filter strips hydrology and sediment transport model: model documentation and user's manual (version 1.04). North Carolina: Biological and Agricultural Engineering - NCSU, 1999. 92 p.

PATHAK, P.; LARYEA, K.B.; SUDI, R. A runoff model for small watersheds in the semi-arid tropics. Transactions of the ASAE, St. Joseph, v.32, n.5, p.1619-24, 1989.

PINTO, F.A. Chuvas intensas no Estado de Minas Gerais: análises e modelos. 1995. 87 f. Tese (Doutorado em Engenharia Agrícola) - Universidade Federal de Viçosa, Viçosa, 1995.

PRUSKI, F.F.; BRANDÃO, V.S.; SILVA, D.D. Escoamento superficial. Viçosa: UFV, 2003. 88 p.

PRUSKI, F.F.; FERREIRA, P.A.; RAMOS, M.M.; CECON, P.R. Model to design level terraces. Journal of Irrigation and Drainage Engineering, Reston, v.123, n.1, p.8-12, 1997.

SCHWAB, G.O.; FREVERT, R.K.; EDMINSTER, T.W.; BARNES, K.K. Soil and water conservation engineering. $2^{\text {nd }}$ ed. New York: John Wiley, 1966. 683 p.

SILVA, J.M.A. Modelo hidrológico para o cálculo do hidrograma de escoamento superficial em bacias hidrográficas: desenvolvimento e aplicação. 2002. 137 f. Tese (Doutorado em Engenharia Agrícola) - Universidade Federal de Viçosa, Viçosa, 2002.

SILVA, M.M.P.C.S.R. Modelo distribuído de simulação do escoamento superficial. 1996. $321 \mathrm{f}$. Tese (Doutorado) - Universidade Técnica de Lisboa, Lisboa, 1996.

SOIL CONSERVATION SERVICE. National engineering handbook.[s.1.:s.n.], 1972. Section 4, Hydrology.

US Department of Agriculture. Soil Conservation Service. National engineering handbook. Washington, 1985. Section 4, Hidrology.

VILLELA, S.M.; MATTOS, A. Hidrologia aplicada. São Paulo: McGraw-Hill, 1975. 245 p. 